\title{
Temperatura basal em idosos asilados
}

Basal body temperature in institutionalized elderly

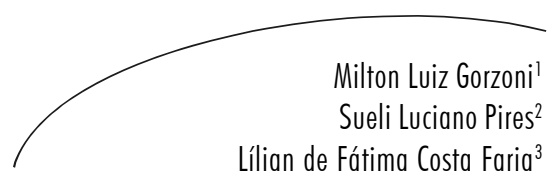

Resumo

Introdução: Idosos tendem a apresentar temperaturas corporais menores do que adultos jovens. A determinação da temperatura basal em populações de instituições de longa permanência para idosos (ILPIs) auxiliará em novas definições sobre estados febris nessa faixa etária e nesses locais, evitando diagnósticos tardios ou equivocados. Objetivo: Determinar valores de temperatura basal axilar em internados clinicamente estáveis em instituição de longa permanência para idosos (ILPI). Casuística e método: Avaliaram-se, em agosto de 2005 , idosos (idade $\geq 60$ anos) de ambos os sexos, com grau de dependência intermediária (perda de duas dentre seis atividades básicas da vida diária) e residentes em ILPI. Utilizaram-se média e desvio padrão de três temperaturas axilares matinais, obtidas por meio de termômetro clínico prismático IncotermÒ. O mês de agosto de 2005 apresentou, na região da ILPI deste estudo, temperaturas ambientais médias entre $14^{\circ} \mathrm{C} \mathrm{e} 26^{\circ} \mathrm{C}$. Resultados: Obtiveram-se dados de 47 idosos (16 mulheres, 31 homens) com média etária de $72,8 \pm 10,6$ anos. Temperatura axilar média matinal: $36,1^{\circ} \pm 0,2^{\circ} \mathrm{C}$, sendo de $36,1^{\circ} \pm 0,2^{\circ} \mathrm{C}$ nas mulheres e de $36,2^{\circ} \pm 0,2^{\circ} \mathrm{C}$ nos homens. Não houve significância estatística quanto ao sexo ou faixa etária e temperaturas aferidas. Conclusão: Idosos institucionalizados apresentam temperatura axilar média matinal e/ou basal inferior à normal em adultos jovens. Este fato merece atenção pelo risco do desenvolvimento de síndromes com temperaturas atípicas como, por exemplo, quadros infecciosos afebris e hipotermias.

\footnotetext{
Departamento de Clínica Médica, Disciplina de Gerontologia da Faculdade de Ciências Médicas da Santa Casa de São Paulo Brasil (FCMSCSP). São Paulo, SP, Brasil.

2 Faculdade de Ciências Médicas da Santa Casa de São Paulo e Hospital Geriátrico e de Convalescentes Dom Pedro II (HGCDPII) da Irmandade da Santa Casa de Misericórdia de São Paulo. São Paulo, SP, Brasil.

3 Unidade de Agudos do Hospital Geriátrico e de Convalescentes Dom Pedro II (HGCDPII) da Irmandade da Santa Casa de Misericórdia de São Paulo. São Paulo, SP, Brasil.
}

Correspondência / Correspondence

Milton Luiz Gorzoni

Hospital Geriátrico e de Convalescentes Dom Pedro II

Av Guapira, 2674

02265-002 - São Paulo,SP, Brasil

E-mail: gorzoni@uol.com.br

Palavras-chave:

Temperatura Corporal. Idoso. Instituição de Longa Permanência para Idosos. 


\section{Abstract}

Introduction: The elderly tend to present lower body temperatures than younger adults. Determining the basal body temperature in long term institutionalized elderly populations will help find new definitions of febrile state for that age group and location, thus avoiding late or wrong diagnosis. Objective: Determine the axillary basal body temperature values in clinically stable elderly living in long term care (LTC) institutions. Method: In August 2005 institutionalized elderly from both genders (age $\geq 60$ years), with intermediate dependence level (loss of two out of six basic daily activities) were evaluated. The average and standard deviation for three morning axillary temperatures was obtained through IncotermÒ prismatic clinical thermometer measurements. In the LTC institution area for the study herein August 2005 has presented average environmental temperatures between $14^{\circ} \mathrm{C}$ and $26^{\circ} \mathrm{C}$. Results: 47 elderly patients were studied (16 women, $31 \mathrm{men}$ ) with an average age of $72.8 \pm 10.6$ years. Morning average axillary temperature: $36.1^{\circ} \pm 0.2^{\circ} \mathrm{C}$, with $36.1^{\circ} \pm 0.2^{\circ} \mathrm{C}$ for women and $36.2^{\circ} \pm$ $0.2^{\circ} \mathrm{C}$ for men. There was no statistical significance related to gender or age and temperatures measured. Conclusion: Institutionalized elderly presented an average or basal morning axillary temperature lower than normal in young adults. That fact deserves attention due to the risk of developing syndromes with atypical temperatures, such as non-febrile infectious profiles and hypothermias.
Key words: Aged. Body Temperature. Homes for the Aged.

\section{INTRODUÇÃO}

O rito e a tradição de avaliar a temperatura de humanos, vivos e mortos, antecedem em muito qualquer evidência científica sobre o conceito de valores normais. Considera-se a temperatura corporal como importante diferencial entre saúde e doença. Sua aferição se tornou parte do exame físico padrão e é fator significativo em muitas decisões diagnósticas e terapêuticas. $O$ conceito de temperatura corporal normal ou basal surgiu em meados do século XIX. Dados apresentados em 1868 por Wunderlich, sobre número superior a um milhão de medidas de temperatura axilar em mais de 25.000 pacientes, definiram $37,0^{\circ} \mathrm{C}$ como temperatura normal para o corpo humano e valores entre $36,3^{\circ} \mathrm{C}$ e $37,5^{\circ} \mathrm{C}$ como extremos da normalidade. Essas graduações de temperatura foram posteriormente associadas ao denominado termômetro clínico utilizado na prática clínica usual. ${ }^{1-3}$ Aceita-se atualmente que a temperatura corporal apresenta oscilações de valores quanto ao sexo e aos horários e locais anatômicos de aferição. Isto provoca dificuldades quanto a definições de normalidade em adultos jovens e principalmente em idosos, notadamente os mais frágeis que compõem a maioria da população residente em instituições de longa permanência para idosos (ILPIs). ${ }^{1,3}$

\section{CASUÍSTICA E MÉTODO}

Selecionaram-se institucionalizados no Hospital Geriátrico e de Convalescentes Dom Pedro II (HGCDPII) da Irmandade da Santa Casa de Misericórdia de São Paulo, com idade igual ou maior a 60 anos, de ambos os sexos, clinicamente estáveis e residentes em unidades classificadas como adequadas para pacientes com dependência intermediária. Conceituou-se como dependência intermediária ou parcial a perda de duas dentre seis atividades básicas da vida diária (banhar-se, vestir-se, uso de sanitário, mover-se em cama e cadeira, continência urinária e fecal, alimentar-se). ${ }^{4}$ Excluíram-se idosos com quadros agudos - infecciosos ou inflamatórios portadores de neoplasias em atividade e/ou medicados com antibióticos, anti-inflamatórios não-hormonais e antipiréticos.

Procurando-se maior precisão sobre a temperatura basal individual, utilizaram-se média e desvio padrão das três primeiras temperaturas matinais, anotadas nas planilhas de sinais vitais 
de cada idoso, durante período climático sem variações extremas de temperatura (agosto de 2005). As tomadas de temperatura seguiram técnica padrão ${ }^{5}$ e foram realizadas pelo corpo de enfermagem responsável pelas unidades. $\mathrm{O}$ instrumento de aferição padrão foi o termômetro clínico prismático Incoterm ${ }^{\circledR}$ (leitura direta, enchimento de mercúrio, escala externa de $+35,0^{\circ} \mathrm{C} \mathrm{a}+42,0^{\circ} \mathrm{C}$, divisão de $0,1^{\circ} \mathrm{C}$, precisão de $\pm 0,1^{\circ} \mathrm{C}$, registro no Ministério da Saúde $n^{\circ}$ 10343200005). O mês de agosto de 2005 apresentou temperatura média compensada entre $14^{\circ} \mathrm{C}$ e $26^{\circ} \mathrm{C}$, segundo a Estação Meteorológica do Mirante de Santana, que, como o HGCDPII, encontra-se situada na região norte da cidade de São Paulo. ${ }^{6}$

Utilizou-se, para testar a significância estatística de diferenças, o teste exato de Fisher quanto às proporções e o teste $t$ de Student em relação às médias. Dividiram-se as casuísticas entre os dois sexos e faixas etárias (60 a 74 anos e igual ou maior de 75 anos de idade), considerando-se estatisticamente significante $\alpha$ de 5,0\%.

O presente trabalho faz parte do Projeto $\mathrm{n}^{\circ} .057 / 04$, aprovado pelo Comitê de Ética em Pesquisa da Irmandade da Santa Casa de Misericórdia de São Paulo.

\section{RESULTADOS}

Obtiveram-se dados de 47 idosos (tabela 1$)$ $72,8 \pm 10,6$ anos de idade (60 a 99 anos) e 36,1 $1^{\circ}$ $\pm 0,2^{\circ} \mathrm{C}$ de temperatura basal matinal axilar (extremos de $35,8^{\circ}$ e $36,8^{\circ} \mathrm{C}$ ). A casuística foi composta por 16 mulheres com idade média de $78,9 \pm 8,2$ anos (67 a 92 anos) e 31 homens com média etária de 69,7 \pm 9,6 anos (60 a 99 anos). Não houve variações significativas de temperatura quanto ao sexo ou à idade, embora a casuística apresentasse maior número de homens com idade igual ou inferior a 74 anos $(p=0,02)$.

Tabela 1 - Características da casuística de idosos institucionalizados e suas temperaturas basais. São Paulo, SP, 2009.

\begin{tabular}{lcccccccc}
\hline Características & \multicolumn{3}{c}{ Mulheres } & \multicolumn{3}{c}{ Homens } & Total & P \\
\hline Faixa etária (anos) & $\leq 74$ & $\geq 75$ & Total & $\leq 74$ & $\geq 75$ & Total & & \\
Número & 5 & 11 & 16 & 22 & 9 & 31 & 47 & 0,02 \\
Idade média (anos) & $69,2 \pm 1,9$ & $83,3 \pm 5,5$ & $78,9 \pm 8,2$ & $64,5 \pm 4,4$ & $82,8 \pm 9,2$ & $69,7 \pm 9,6$ & $72,8 \pm 10,6$ & NS \\
Temperatura & & & & & & & & \\
matinal média $\left({ }^{\circ} \mathrm{C}\right)$ & $35,9 \pm 0,3$ & $36,1 \pm 0,1$ & $36,1 \pm 0,2$ & $36,2 \pm 0,2$ & $36,2 \pm 0,2$ & $36,2 \pm 0.2$ & $36,1 \pm 0,2$ & NS \\
\hline
\end{tabular}

NS= não significante

\section{DISCUSSÃO}

Idosos necessitam de aferições periódicas de sua temperatura basal. Deve-se ter este cuidado corriqueiro pela predisposição, relacionada ao envelhecimento, do desenvolvimento de síndromes com temperaturas atípicas como, por exemplo, quadros infecciosos afebris e hipotermias. ${ }^{7-9}$ Isto decorre do encontro, frequente na terceira idade, de fatores relacionados a temperaturas basais baixas - diabetes mellitus, doenças neurológicas, desnutrição, sarcopenia, imobilidade e medicamentos (barbitúricos, opióides, antidepressivos tricíclicos, benzodiazepínicos, fenotiazidas e alfa-bloqueadores) - situações comuns em ILPIs. ${ }^{8,10,11}$

Locais anatômicos de aferição, horários e ambientes propiciam, além da idade, variações de valor sobre temperatura corporal normal. A 
literatura consultada relata que, enquanto a temperatura oral matinal em adultos jovens gira em torno de $37,2^{\circ} \mathrm{C}$, em idosos saudáveis e residentes em domicílios sua média é entre $36,8^{\circ} \mathrm{C}$ e 36, $9^{\circ} \mathrm{C} \cdot{ }^{1,12-14}$ Idosos hospitalizados e sem doenças febris apresentam temperatura retal entre $37,2^{\circ} \mathrm{C}$ e $37,4^{\circ} \mathrm{C}$ e axilar em torno de $36,3^{\circ} \mathrm{C} . .^{15,16} \mathrm{~A}$ temperatura oral matinal em idosos institucionalizados oscila entre $36,2^{\circ} \mathrm{C}$ e $36,5^{\circ} \mathrm{C} .17,18$

Circunstâncias e doenças comuns durante a terceira idade favorecem questionamentos sobre praticidade e riscos de resultados errados em todas as técnicas de medida de temperatura corporal. ${ }^{19}$ Estados confusionais, xerostomia, respiração bucal e falhas de dentição dificultam o posicionamento estável de termômetros na cavidade bucal. ${ }^{20}$ Não concordância do examinado e variações de profundidade em inserções retais de termômetros tornam difícil o uso cotidiano dessa via em idosos. $^{20}$ Mensurações de temperatura relacionadas ao aparelho auditivo podem apresentar variações de até $0,5^{\circ} \mathrm{C}$, pela presença de rolha de cerúmen. ${ }^{20,21}$

A temperatura na região axilar também sofre interferências devido a dobras na pele, espessura da camada adiposa subcutânea, massa muscular em membro superior dominante, distúrbios circulatórios regionais e temperatura ambiental. ${ }^{20}$ Mas, pela tradição e praticidade, é o local mais aceito e utilizado na prática clínica brasileira, razão de sua escolha como ponto de aferição da temperatura dos idosos neste estudo. Merece menção o fato que, na literatura consultada, poucos foram os estudos em idosos encontrados sobre este sítio de mensuração de temperatura. Seus resultados relatam média de $36,17^{\circ} \pm$ $0,21^{\circ} \mathrm{C}$, extremos de $35,5^{\circ}$ e $37,0^{\circ} \mathrm{C}$ e queda de $0,15^{\circ} \mathrm{C} /$ década de vida na temperatura basal média. ${ }^{15,22,23} \mathrm{O}$ presente estudo encontrou média e desvio-padrão semelhantes aos do estudo de Gubin et al. ${ }^{22}$ (2006) e seus extremos de temperatura próximos aos de Darowski et al. ${ }^{15}$ (1991). O não-encontro, na presente casuística, da redução de $0,15^{\circ} \mathrm{C} /$ década de vida na temperatura basal média, pode ser decorrente do seu número de idosos (47), do local de avaliação
(ILIP) e dos critérios de exclusão. Cabe também a observação de que Roghmann et al. ${ }^{23}$ (2001) estudaram 320 pacientes, retrospectivamente, utilizando modelo estatístico de regressão linear, durante e após tratamento hospitalar por pneumonia. Mesmo assim, seguindo a regra proposta por esses autores ${ }^{23}$ e considerando-se temperaturas axilares normais em torno de $36,8^{\circ} \mathrm{C}$ aos 20 anos de idade, ${ }^{1,3,20}$ o valor estimado para 70 anos de idade seria de $36,8^{\circ}$ - 0,75 (5 décadas de vida $\left.x 0,15^{\circ}\right)=36,05^{\circ} \mathrm{C}$, próximo ao observado neste estudo.

Desde a pesquisa inicial de Wunderlich, em 1868, tem-se utilizado o termômetro de mercúrio como "padrão ouro" para aferição de temperaturas corporais, razão de seu uso no presente estudo. Há, porém, ações internacionais e locais procurando reduzir e/ou retirá-lo da prática diária dos profissionais da saúde. Isto decorre do risco de envenenamento pelo vapor de mercúrio formado durante quebras acidentais de termômetros. Fica, porém, a observação de que não há diferenças significativas nos valores medidos entre os termômetros convencionais e os de tecnologia mais recente. ${ }^{20,21,24}$

Pesquisa no portal PubMed (http:// www.nlm.nih.gov) utilizando os unitermos Brazil, nursing home, elderly encontrou 11 artigos, nenhum relacionado a aferições de temperatura corporal. No mesmo portal e na Scielo - Scientific Electronic Library Online (http://www.scielo.br), busca com os unitermos axillary, temperature, elderly apresentou ausência de citações. Tal fato também foi observado com os unitermos nursing home, temperature, elderly no segundo portal citado. O que a literatura consultada relata é a tendência universal de menores temperaturas corporais durante o processo do envelhecimento, independentemente do local de aferição e principalmente no segmento de idosos residentes em ILPIs, com resultados semelhantes aos obtidos no presente estudo. Justifica-se esta observação pelo padrão de doenças, medicações e graus de nutrição e dependência física em suas casuísticas de institucionalizados. ${ }^{7,17,18,22,23}$ Cabe, assim, a proposta de Yoshikawa e Norman ${ }^{25}$ sobre critérios de definição de febre em idosos: (1) aumento 
persistente da temperatura corporal em pelo menos $2^{\circ} \mathrm{F}\left(1,1^{\circ} \mathrm{C}\right)$ sobre valores basais, independentemente da técnica de aferição (oral, retal, axilar ou auricular); (2) temperaturas orais iguais ou maiores a $99^{\circ} \mathrm{F}\left(37,2^{\circ} \mathrm{C}\right)$ e $(3)$ temperaturas retais iguais ou maiores a $99,5^{\circ} \mathrm{F}$ $\left(37,5^{\circ} \mathrm{C}\right)$.

Conclui-se, desta forma, que a presente casuística de idosos institucionalizados apresentou temperatura axilar basal matinal inferior à normal em adultos jovens. Este fato merece atenção pelo risco do desenvolvimento de síndromes com

\section{REFERÊNCIAS}

1. Mackowiak PA, Wasserman SS, Levine MM. A critical appraisal of 98.6 degrees $F$, the upper limit of the normal body temperature, and other legacies of Carl Reinhold August Wunderlich. JAMA 1992 sep; 268(12): 1578-80.

2. Pearce JM. A brief history of the clinical thermometer. QJM. 2002 Apr; 95(4): 251-2.

3. Sund-Levander M, Forsberg C, Wahren LK. Normal oral, rectal, tympanic and axillary body temperature in adult men and women: a systematic literature review. Scand J Caring Sci 2002 jun; 16(2): 122-8.

4. Katz $\mathrm{S}$, et al. Progress in development in the index of ADL. Gerontologist 1970 apr; 10(1): 20-30.

5. Mallari B. Temperature. University of Illinois Medical Center: Health Library. disponível em URL: http://uimc.discoveryhospital.com/ main.php?id $=2954$

6. Instituto Nacional de Meteorologia Temperaturas diárias. disponível em URL: http://reia.inmet.gov.br/graficos/ ss00200400383781200508.gif

7. Gomolin IH, et al. Older is colder: temperature range and variation in older people. J Am Geriatr Soc 2005 dec; 53(12): 2170-2.

8. Kenney WL, Munce TA. Invited review: aging and human temperature regulation. J Appl Physiol 2003 dec; 95(6): 2598-603

9. Van Someren EJ. Thermoregulation and aging. Am. J. Physiol Regul. Integr. Comp Physiol 2007 jan; 292(1): R99-102.

10. Gorzoni ML, Pires SL. Idosos asilados em hospitais gerais. Rev Saude Publica 2006 dec; 40(6): 1124-30. temperaturas atípicas. A determinação da temperatura basal em populações de ILPIs auxiliará em novas definições sobre estados febris nessa faixa etária e nesses locais, evitando diagnósticos tardios ou equivocados.

\section{AGRADECIMENTO}

Agradecemos ao Núcleo de Apoio à Publicação da Faculdade de Ciências Médicas da Santa Casa de São Paulo - NAP-SC, pelo suporte técnico-científico à publicação deste manuscrito.
11. Mallet ML. Pathophysiology of accidental hypothermia. QJM 2002 dec; 95(12): 775-85.

12. Eriksson $\mathrm{H}$, et al. Body temperature in general population samples. The study of men born in 1913 and 1923. Acta Med Scand 1985; 217(4): 34752.

13. Marion GS, McGann KP, Camp DL. Core body temperature in the elderly and factors which influence its measurement. Gerontology 1991; 37(4): 225-32. 14. McGann KP, et al. The influence of gender and race on mean body temperature in a population of healthy older adults. Arch Fam Med 1993 dec; 2(12): 1265-7.

14. Darowski A, Weinberg JR, Guz A. Normal rectal, auditory canal, sublingual and axillary temperatures in elderly afebrile patients in a warm environment. Age Ageing 1991 mar; 20(3): 113-9.

15. Smitz $\mathrm{S}$, et al. Comparison of rectal and infrared ear temperatures in older hospital inpatients. J Am Geriatr Soc. 2000 jan; 48(1): 63-6.

16. Castle SC, et al. Fever response in elderly nursing. J Am Geriatr Soc 1991 sep; 39(9): 853-7.

17. Gomolin IH, Lester P, Pollack S. Older is colder: observations on body temperature among nursing home subjects. J Am Med Dir Assoc 2007 jun; 8(5): 335-7.

18. Darowski A, et al. The febrile response to mild infections in elderly hospital inpatients. Age Ageing 1991 may; 20(3): 193-8.

19. Smith LS. Reexamining age, race, site, and thermometer type as variables affecting temperature measurement in adults - $\mathrm{A}$ comparison study. BMC Nurs 2003 jun; 2(1): 1. 
20. Sund-Levander M, et al. Errors in body temperature assessment related to individual variation, measuring technique and equipment. Int J Nurs Pract 2004 oct; 10(5): 216-23.

21. Gubin DG, et al. The circadian body temperature rhythm in the elderly: effect of single daily melatonin dosing. Chronobiol Int 2006 nov; 23(3): 639-58.
22. Roghmann MC, Warner J, Mackowiak PA. The relationship between age and fever magnitude. Am J Med Sci 2001 aug; 322(2): 68-70.

23. Davies SP, et al. A comparison of mercury and digital clinical thermometers. J Adv Nurs 1986 sep; 11(5): 535-43.

24. Yoshikawa TT, Norman DC. Fever in the elderly. Infect Med 1998; 15(10): 704-8.

Recebido: $21 / 8 / 2009$

Aprovado: 19/1/2010 